\title{
USO DE INDICADORES NA GESTÃO DE RECURSOS DE INFORMAÇÃO
}

\author{
Helen Beatriz Frota Rozados
}

\begin{abstract}
Resumo
Aborda aspectos de mensuração, focalizando o uso de instrumentos de medição, particularmente indicadores, para a avaliação de recursos de informação. Conceitua indicadores de desempenho, salientando a importância de seu uso na gestão destes recursos, com foco no cliente/usuário. Comenta sua autoridade para a gestão da qualidade e processo de tomada de decisão. Apresenta documentos que tratam de indicadores de desempenho voltados a bibliotecas tradicionais e digitais, como as Normas Internacionais ISO 11620, ISO 20983, o Projeto EQUINOX, além de um manual publicado pela UNESCO, como fontes básicas e qualificadas para o estudo e a seleção de indicadores e metodologias. Conclui, ressaltando a importância do uso sistemático de indicadores para a tomada de decisão, avaliação e gestão de recursos de informação. Recomenda a promoção de ações que visem sensibilizar órgãos de pesquisa e organismos governamentais para o incentivo a sua adoção, bem como a implementação de conteúdos sobre o assunto, junto aos cursos da área de Ciência da Informação, dentro do processo de desenvolvimento de competência em informação.
\end{abstract}

Palavras-chave

Indicadores de desempenho; Recursos de informação; Unidades de informação; Serviços de informação; Abordagem centrada no usuário; Competência em informação.

\section{THE USE OF INDICATORS FOR INFORMATION RESOURCES MANAGEMENT}

\begin{abstract}
It approaches measurement aspects, focusing the measurement instruments use, particularly indicators, to information resources evaluation. It conceptualizes performance indicators, explaining how important it is to use it on managing these resources, focused on the client/user. It comments its authority for quality management and decision taking. It presents documents deal with performance indicators concerning traditional and digital libraries, among which International Standards ISO 11620, ISO 20983, the EQUINOX Project, plus a manual published by UNESCO, as basic and qualified sources for the formation and selection of indicators and methodologies. It concludes, highlighting the importance of the systematic use of indicators for evaluation, decision taking and information resources management. It recommends the promotion of actions aiming to motivate research and governmental agencies to adopt them, and also the inclusion of studies on the subject in graduate courses, in the field of Information Science, in the process of developing competence in information.
\end{abstract}

\section{Keywords}

Performance indicators; Information resources; Information units; Information service; User-centered approach; Competence in information. 


\section{A MÉTRICA E OS SISTEMAS DE MENSURAÇÃO}

Willian Thompson - Lorde Kelvin (1824-1907), conceituado físico que deu fundamental contribuição à termodinâmica, costumava dizer: "Se algo não pode ser medido, ele realmente não existe". Medir objetos e eventos não é apenas uma necessidade científica, mas é dar significado à complexidade dos fenômenos naturais. Medir é uma atividade rotineira do ser humano e de sua interação com a natureza. Para que se possa realizar medições é condição fundamental a existência de métricas e instrumentos de trabalho adequados.

Segundo Geisler (2000), fenômenos físicos como temperatura, peso, massa, são adequadamente medidos, em geral, com o uso de um simples aparelho. O mesmo autor também afirma que as Ciências Sociais, Administrativas e Comportamentais são fenômenos considerados muito menos precisos, dos quais, muitas vezes, possuímos pouco conhecimento, o que os torna muito mais difíceis de medir. O que medir é, portanto, o primeiro princípio da mensuração.

Uma vez decidido o que medir, passa-se a definir que instrumento de medida pode dar conta do que se deseja medir. Instrumentos de mensuração têm uma variedade de características que impactam sua eficiência.

Geisler (2000) identifica que o processo de seleção de uma métrica é influenciado por três fatores: a cultura da organização; um conjunto disponível de métricas e o tipo de atividade a ser medida; e outras influências, como os atores envolvidos (comunidade científica e de negócios ou interesses governamentais). A definição de métrica inclui três aspectos: o item medido (o que medir), a unidade de medida (como medir) e o inerente valor associado com a métrica (por que medir ou o que se pretende encontrar com esta mensuração). Afirma, também, que para avaliar ciência e tecnologia existe um único método viável: mensuração por indicadores. Torna a enfatizar que múltiplos indicadores são necessários para proporcionar uma adequada cobertura de dimensões e aspectos de processos complexos, atividade e resultados. Um indicador é uma medida reservada para a descrição ou representação de um dado evento ou fenômeno. Uma métrica pode conter um ou mais indicadores. 


\section{INDICADORES}

O conceito de indicadores pouco varia de um autor para outro ou deles para o conceito dado por Geisler (2000). A Financiadora de Estudos e Projetos (FINEP), organização governamental que avalia e gerencia estudos e projetos e fornece consultoria, utiliza maciçamente indicadores e entende que eles são: "Especificação quantitativa e qualitativa para medir o atingimento de um objetivo" (FINEP, 2002). A OCDE, organização internacional com reconhecidas e adotadas publicações sobre o assunto, em uma definição clássica (OCDE, 2001), especifica que indicadores são “[ . . .] uma série de dados definidos para responder perguntas sobre um fenômeno ou um sistema dado". A International Standart Organization (ISO) estabelece que indicadores são: "Expressão (numérica, simbólica ou verbal) empregada para caracterizar as atividades (eventos, objetos, pessoas), em termos quantitativos e qualitativos, com o objetivo de determinar o valor" (ISO, 1998, p. 3).

Observa-se que as idéias: medida, qualitativo e quantitativo são constantes nos conceitos aqui apresentados, o que leva a perceber que indicadores nada mais são do que unidades que permitem medir - caso de elementos quantitativos, ou verificar - caso de elementos qualitativos, se estão sendo alcançados os objetivos ou as mudanças previstas. Também possibilitam conhecer melhor os avanços em termos de resultados ou de impactos. Um indicador é, portanto primordialmente, uma ferramenta de mensuração, utilizada para levantar aspectos quantitativos e/ou qualitativos de um dado fenômeno, com vistas à avaliação e a subsidiar a tomada de decisão.

Com relação às características que são importantes a um conjunto de indicadores, com vistas a garantir a validade dos dados coletados, Martinez et al (1998) sugerem: generalidade; possibilidade de correlação entre as distintas variáveis ou os diferentes contextos; temporalidade. Francisco et al (2000?) salientam que toda a avaliação parte de um princípio de comparação, sendo, portanto, necessário que haja dados passíveis de serem comparados, que permitam ser coletados de forma semelhante em todos os casos. As colocações de Martinez et al (1998) e de Francisco et al (2000?), refletem as mesmas preocupações, uma vez que a generalidade, a possibilidade de correlação e a temporalidade - que visa estabelecer as séries estatísticas - são aspectos que permitirão a elaboração de dados e informações com potencial para comparações. 
Além de serem um instrumento de avaliação e exatamente por isto, os indicadores também são instrumentos de gestão. A gestão implica capacidade de operar sobre dimensões-chave de sistemas e de processos distintos, modificando seus estados e seus rumos (ALBORNOZ et al, 1997).

$\mathrm{O}$ requisito para que os indicadores sejam úteis à gestão implica, necessariamente, que estejam normalizados e que sua produção histórica (temporalidade) se atenha sempre à mesma norma ou forma de medida, a fim de permitir a comparabilidade.

Para poder eleger indicadores deve-se ter uma concepção precisa das organizações e sistemas a serem gerenciados, fato este que pode apresentar diferentes níveis de facilidade ou complexidade. Portanto, diferentes tipos de indicadores existem para que adequações a organizações, a objetivos, a metas e à missão possam ser efetuadas, permitindo medir diferentes tipos de atividade ou atingir objetivos diversos.

Importante aspecto a ser observado é que, para que os indicadores sejam ferramentas úteis, devem ser produzidos com regularidade, visando à formação de séries temporais e permitindo visualizar as tendências no tempo e nos dados, além de possibilitar comparações internacionais. Além do mais, os dados precisam estar disponíveis para um público amplo e de forma acessível.

\subsection{INDICADORES DE DESEMPENHO E OS SISTEMAS E SERVIÇOS DE INFORMAÇÃO}

$\mathrm{Na}$ área de Ciência da Informação, assim como ocorre em outras áreas do conhecimento, os autores Briand et al (2002), Sutter (2002) e Moore (1989) preocuparam-se em estabelecer classificações para os indicadores. Diferentemente de Geisler (2000) que classifica os indicadores em duas grandes dimensões - de ciência e social, Briand et al (2002) os separam em dois grandes grupos: indicadores quantitativos e indicadores qualitativos. Por indicadores quantitativos os autores entendem aqueles que podem ser definidos por uma unidade de contagem. Já os indicadores qualitativos são os que se referem à preocupação com a avaliação e a pesquisa de qualidade. Neste caso, eles podem ser diretos ou indiretos. São diretos quando estão ligados aos três componentes da pesquisa de informação: a qualidade e o número de fontes de informação escolhidas; o valor agregado pelo trabalho do profissional da informação; a satisfação do usuário. São 
indiretos quando representam a medida da notoriedade. Deste modo, o número de clientes é um indicador quantitativo bruto da freqüência dos serviços documentários, mas quando desmembrado em novos clientes e clientes regulares, torna possível elaborar indicadores qualitativos indiretos relativos à notoriedade dos serviços.

Dividindo os indicadores nesses dois grandes grupos Briand et al (2002) propõem um conjunto de indicadores de desempenho para serviços documentários. A proposta tem o foco no usuário-cliente, de acordo com a tônica das atuais avaliações de serviços.

Para Sutter (2002), múltiplos indicadores podem ser utilizados por uma organização: uns para resolver problemas conjunturais, outros para assegurar que os procedimentos internos sejam aplicados; outros, ainda, para levantar aspectos econômicos da organização, qualidade de seus produtos, satisfação de sua clientela. Com relação à satisfação de usuários e qualidade de serviços e produtos, o autor os subdivide em dois outros tipos: os indicadores de satisfação e os indicadores de desempenho.

Indicadores de satisfação dos usuários podem se apoiar na avaliação de um ou vários serviços ou produtos, sobre uma ou várias características. Para uma unidade de informação, os indicadores de satisfação dizem respeito a aspectos como horários de abertura, número de lugares disponíveis para leitura, pesquisa, consulta em terminais de computador, disponibilidade de documentos, serviços de empréstimo, serviço de empréstimo entre bibliotecas, serviço de referência, comportamento do pessoal, ergonomia dos catálogos on-line, sinalização do acervo ou referir-se a uma apreciação mais global.

Para esse autor, a construção de um indicador de satisfação, poderá ser feito a partir dos resultados de enquetes periódicas, baseadas num questionário que permita levantar o grau de satisfação e o grau de importância atribuída a uma dada prestação de serviço ou característica do serviço ou produto.

Os indicadores de desempenho buscam medir a eficiência e a eficácia de uma unidade de informação. Ao se pensar em termos de aquisição e fornecimento de documentos, os indicadores de desempenho tendem a medir a rapidez do fornecimento (eficiência), a exatidão do fornecimento (eficácia), o custo unitário do fornecimento (custos), o número de documentos disponibilizados para empréstimo num determinado período (produtividade). 
Moore (1989), um dos primeiros estudiosos a se aprofundar em medidas de desempenho para bibliotecas, subdivide os indicadores em de recursos - uma vez que o volume de recursos de uma biblioteca determina o limite de sua capacidade - e indicadores de produtos, que irão medir os produtos gerados pelos recursos utilizados pelos serviços da biblioteca. A definição do conjunto total dos indicadores por ele sugerido parte de oito princípios fundamentais, ainda pertinentes e atuais para qualquer tipo de unidade ou serviço de informação, não obstante terem sido propostos para bibliotecas públicas. São eles:

a) a avaliação deve fazer parte integrante do processo de gestão;

b) a eficácia deve ser avaliada em função das necessidades dos consumidores;

c) a avaliação deve levar em conta os limites impostos pelos recursos disponíveis;

d) a eficácia de uma biblioteca deve ser medida em função de seus objetivos;

e) as bibliotecas públicas oferecem uma gama de serviços diversificados;

f) o nível de avaliação deve ser determinado pelo grau de detalhamento pretendido;

g) a comparação é um elemento essencial à avaliação;

h) os indicadores de base devem ser adaptados às condições locais.

Os autores citados permitem observar que há um consenso, apontando claramente para o uso específico de indicadores de desempenho, incluindo-se neles o de satisfação do cliente/usuário, para avaliação e gestão de unidades de informação e, por extensão, de serviços de informação (BRIAND et al, 2002; MOORE, 1989; SUTTER, 2002). Entendese esta posição solidificada, uma vez que a relevância em se medir desempenho é inquestionável, dado o marcante crescimento e desenvolvimento técnico e econômico ocorrido em décadas recentes, aliado à importância, cada vez mais acentuada, de qualificar e adequar produtos e serviços, sem perder de vista a satisfação do cliente/usuário, fim maior de qualquer serviço de informação.

Sutter (2002) propõe quatro critérios para escolha de indicadores de desempenho. O primeiro refere-se à pertinência, ou seja, um indicador deve ser fiel, deve estampar uma imagem fiel de um fenômeno estudado, com o mínimo de distorção; deve ser justo ou estável, o que significa dar uma informação exata e renovável, e também deve ser preciso ou sensível, indicando que as variações significativas do fenômeno precisam ser refletidas pelas variações coerentes do indicador. O segundo refere-se ao caráter operacional, no qual um indicador deve ser fácil de estabelecer, fácil de utilizar, vendável ou aceitável, ou 
seja, não deve ser contrário à cultura dos destinatários; deve, também, ser comunicante, o que significa permitir o diálogo entre diferentes populações. Como terceiro critério cita o caráter consolidável (agregável) que se refere ao fato de que um indicador deve ser confiável, consolidável, agregável ou acumulável, ou seja, sua consolidação facilita as análises e as sínteses anteriores e permite deixar em evidência as tendências, enquanto durem. O último aspecto diz respeito ao caráter econômico, que significa que um indicador deve ser calculável monetariamente ou ser útil à prevenção.

Os critérios apresentados por Sutter (2002) estão em consonância com as características a serem observadas nos instrumentos de mensuração e os critérios a serem adotados em sua escolha, propostos por Geisler (2000). A estes critérios devem ser agregadas características específicas de serviços de informação, algumas, inclusive próprias da organização a que esses serviços estão vinculados, as quais permitirão definir um conjunto apropriado de indicadores, adequados à mensuração e à avaliação que se deseja realizar.

Percebe-se, em nível mundial, que é cada vez mais crescente o incentivo pela busca da qualidade total nas unidades de informação, fazendo com que aumente a preocupação com a definição e a utilização de indicadores apropriados, que auxiliem na avaliação e na gestão destes serviços. Os indicadores de desempenho, por medirem eficiência e eficácia, fatores que estão diretamente ligados à satisfação do usuário-cliente, têm sido os escolhidos por unanimidade, entre os autores e pesquisadores do tema. Concorda-se com esta escolha por se crer que o fim maior da gestão da qualidade é a satisfação do usuário-cliente e que a excelência desta satisfação só pode ser alcançada através de serviços eficientes e eficazes. Por outro lado, a busca pela excelência exige o sistemático levantamento de dados e informações, com a finalidade não somente de avaliar os serviços, mas, principalmente, utilizá-los como elementos do planejamento estratégico. Nesta perspectiva, entendem-se indicadores de desempenho como uma ferramenta para mensurar a satisfação do usuário e a qualidade dos serviços, com vistas à avaliação e à tomada de decisão.

Como se pode observar pelo exposto, o uso de indicadores para avaliação de recursos de informação tem sido uma preocupação em sistemas e serviços de informação documental em nível mundial. 


\subsection{NORMALIZAÇÃO INTERNACIONAL SOBRE INDICADORES EM UNIDADES DE INFORMAÇÃO}

A preocupação com a avaliação de recursos de informação documental, em nível internacional, pode ser verificada através da literatura disponível. Constata-se, através de levantamento bibliográfico utilizando-se ferramentas de busca na Internet (Google, Google Scholar, Mozila) e em bases de dados bibliográficos como a Library Information Science Abstracts (LISA), que o número de publicações sobre avaliação de unidades de informação e, especialmente, a utilização de indicadores para proceder esta avaliação, tem aumentado gradativa e geometricamente, a partir do início dos anos noventa.

Observa-se que esta tendência acentua-se no momento em que entra em ação pelo menos um dos seguintes fatores: em âmbito mundial, as ações de controle e certificação de qualidade, especialmente após o advento da série ISO 9000, se intensificam nas organizações e, conseqüentemente, atingem os serviços de informação a elas vinculados; em âmbito local, a formação do bloco econômico europeu - representado pela União Européia - exigiu a busca pela equiparação econômica, social e cultural. Um dos principais caminhos encontrados foi a disponibilização facilitada de informação, à Comunidade. Para propiciar esta disponibilização, as ações políticas da União Européia voltaram-se para a busca do nivelamento das bibliotecas entre si, especialmente as públicas. Para tal, incentivaram o uso de indicadores de desempenho com o intuito de avaliá-las e, posteriormente, equipará-las. Este incentivo ao uso de indicadores, hoje, vem extrapolando o aspecto do objetivo inicial de equiparação para se tornar um processo contínuo de desenvolvimento e agregação de valores.

A combinação destes dois fatores torna o ambiente propício à elaboração e à publicação da Norma Internacional ISO 11620 - Indicadores de Desempenho para bibliotecas tradicionais (ISO, 1998, 2003a) e, posteriormente, da Norma Internacional ISO 20983 - Indicadores de Desempenho para bibliotecas eletrônicas (ISO, 2003b). Salienta-se aqui o fato de que, após o surgimento da Norma Internacional ISO 11620, ficou muito claro o fato de que a referida norma não atendia as exigências impostas por uma nova ordem de bibliotecas: as bibliotecas eletrônicas. Para amenizar este problema, a União Européia auspiciou o denominado Projeto EQUINOX (EQUINOX, 1999?; BROPHY et al, 2000; BROPHY, 2000?; BROPHY et al, 2001). 
O propósito do projeto foi desenvolver um conjunto de indicadores de desempenho para serviços de bibliotecas eletrônicas, com ênfase especial no acesso à informação e na entrega, nos custos e na satisfação do usuário. A proposta final residia em oferecer um conjunto de indicadores que pudessem realçar e complementar os indicadores para serviços tradicionais de bibliotecas, propostos pela ISO 11620, ao mesmo tempo em que recomendava a utilização unificada dos dois conjuntos de indicadores.

O Projeto baseou-se na definição de qualidade, aceita mundialmente desde os anos 50, que postula que 'a qualidade é a atitude para alcançar o objetivo'. O que significa que a qualidade converte-se em um conceito significativo só quando está ligada ao objetivo de obter a satisfação total do cliente.

Como produtos finais, o projeto apresentou um conjunto de indicadores híbridos normalizados, do funcionamento da biblioteca; um marco híbrido, também normalizado, da gerência da qualidade da biblioteca; um sistema de ajuda estruturado para a tomada de decisão; uma interface aberta visando à coleta dos dados gerados em ambiente eletrônico. Como subproduto, apresentou uma ferramenta de software que pretende ser uma medida de funcionamento e um sistema de gerência da qualidade para bibliotecas, permitindo aos gerentes das bibliotecas selecionar os indicadores que mais se ajustem aos objetivos de suas unidades de informação. Conforme se postulou anteriormente, este Projeto antecedeu a Norma Internacional ISO 20983, que visou suprir esta deficiência, sem, no entanto, apresentar a ferramenta de software que o Projeto propôs, razão pela qual se considerou importante comentá-lo neste texto.

As Normas ISO 11620 e ISO 20983, complementares, surgem, então, dentro da filosofia de gestão da qualidade e da abordagem centrada no usuário, propondo indicadores de desempenho para avaliar bibliotecas tradicionais e eletrônicas. (ISO 1998, ISO 2003a, ISO 2003b). Seus objetivos básicos são: encorajar o emprego de indicadores de desempenho nas bibliotecas tradicionais e eletrônicas e tornar conhecida as formas para medir desempenho. O corpo da Norma define:

a) domínio de aplicação;

b) referência normativa;

c) lista de definições de termos técnicos utilizados; 
d) critérios e quadro de descrição, indicando como critérios: conteúdo informativo, confiabilidade, validade, adequação, aplicabilidade e comparabilidade; e determinando os seguintes pontos no quadro de descrição: nome do indicador, objetivo, domínio de aplicação, fontes, indicadores associados;

e) emprego dos indicadores de desempenho, estabelecendo entorno e dicas para a escolha dos indicadores;

f) limites, definindo a otimização dos resultados, o grau de precisão, competências dos usuários e desempenho das bibliotecas, relação entre os recursos e os serviços, comparabilidade dos dados.

Outro documento importante, abrangendo indicadores, mas com foco em Benchmarking e Melhores Práticas (ou Boas Práticas, como também é utilizado na literatura) é o intitulado 'Guidelines for the Application of Best Practice in Australian University Libraries; Intranational and International Benchmarks' (WILSON et alii, 2000). O documento produzido, sob os auspícios do Council of Australian University Libraries (CAUL) e publicado em 2000, teve como base uma pesquisa sobre as melhores práticas em atividades de bibliotecas acadêmicas e alguns centros de pesquisa australianos. No contexto do trabalho, as 'best practice activitie' abrangem a implementação da estruturação da qualidade, o uso do benchmarking e a medição de desempenho como ferramentas para a melhoria contínua de produtos, processos e serviços. A pesquisa buscou, portanto, propor as melhores práticas para a gestão da qualidade baseadas em um conjunto de indicadores que medem o desempenho das unidades de informação das bibliotecas universitárias australianas e facilitam a prática do benchmarking. $\mathrm{O}$ alvo aqui foi permitir a comparação da excelência entre bibliotecas dos diferentes países da Austrália, a partir da padronização destas práticas.

O produto final oferece recomendações e políticas para a gestão da qualidade, benchmarking e uso de indicadores de desempenho. Com relação aos indicadores, especificamente, eles estão apresentados em forma de tabela, da qual consta o nome do indicador e a fonte de onde foi retirado e o documento final disponibilizado na Web, em http://www.dest.gov.au/archive/highered/eippubs/eip00_11/00_11.pdf.

Nesta breve análise de alguns documentos significativos sobre indicadores para unidades de informação, não se poderia deixar de citar um menos atual, mas não menos importante - 
o projeto de manual, intitulado 'Comment Mensurer l'Efficacité des Bibliothèques Publiques' desenvolvido por Nick Moore, sob os auspícios de Programme General d'Information et UNISIST, da UNESCO. O documento final foi publicado em Paris, em 1989 (MOORE, 1989). Seu valor está no fato de ter embasado os estudos posteriores desenvolvidos sobre o assunto, sendo constantemente citado e mantendo a atualidade de seu conteúdo.

O trabalho tem a preocupação de conceituar e/ou definir os termos adotados, principalmente tendo em vista as enormes diferenças de natureza e de organização, encontrada nas bibliotecas dos diferentes países. Propõe indicadores que se situam em dois níveis. O primeiro corresponde a uma abordagem global geral, em escala de um país ou de um sistema de serviços de biblioteca. O segundo diz respeito a uma avaliação mais detalhada para medir a eficácia de uma biblioteca, vista individualmente.

Um dos capítulos explica como efetuar as sondagens de opinião junto aos consumidores (usuários), nele é apresentado, como instrumento, um questionário com perguntas abertas e fechadas. Dois outros capítulos estão consagrados à adaptação dos indicadores e à interpretação dos resultados. Os indicadores estão divididos em grandes grupos (recursos, produtos e opinião dos usuários) e, dentro destes grupos, são apresentados por níveis. Cada indicador traz as seguintes informações: nome do indicador, objetivos, dados, notas e cálculo. Igual estrutura será, posteriormente, adotada tanto na Norma ISO 11620 (ISO, 1998, 2003a), Norma ISO 20983 (ISO, 2003b) quanto no Projeto EQUINOX (BROPHY et al, 2000). Ao tratar da adaptação dos critérios de avaliação, o autor comenta que é impossível existir um manual de avaliação que seja instantaneamente aplicável a qualquer biblioteca sugerindo o estudo de caso como uma técnica para adequar o manual proposto à prática necessária a cada biblioteca.

\subsection{SELEÇÃO E CRITÉRIOS PARA A APLICAÇÃO DE INDICADORES}

Ao selecionar indicadores para avaliar unidades de informação, incluindo-se aqui os propostos pelas Normas Internacionais ISO 11620 e ISO 20983, Baba (2003) aponta que é importante levar-se em consideração alguns elementos referentes à organização a qual os indicadores serão aplicados. São eles:

a) a missão;

b) as metas e os objetivos; 
c) o ambiente externo e seus impactos;

d) a infra-estrutura de serviços;

e) os processos que contribuem para a realização das metas e dos objetivos - incluem atividades e construção de competências;

f) revisão dos indicadores;

g) dados de base - coletados onde o serviço é feito;

h) alvos baseados nos dados de base;

i) implementação e monitoramento.

Verifica-se que os elementos listados assinalam semelhanças encontradas nos oito princípios fundamentais propostos por Moore (1989), cerca de quatorze anos antes, relacionados no item 2.1 Indicadores de Desempenho e os Sistemas e Serviços de Informação, deste documento. Esses elementos referem-se às características do sistema ou serviço, o que se está tentando alcançar, às atividades a serem observadas e às competências que precisam ser construídas. Além disto, apontam para o fato de que os indicadores não são permanentes ou imutáveis e que, portanto, devem ser revistos. Abalizam que os dados coletados e que irão compor a base das informações, precisam ser recolhidos onde o serviço é feito, ressaltando que os alvos escolhidos deverão estar fundamentados nos dados de base. Conclui, sinalizando a importância de implementar e monitorar, constantemente, o processo de medição.

Baba (2003) sugere dois tipos de indicadores: para avaliar serviços aconselha utilizar indicadores de desempenho; para o ambiente externo e seus impactos, indicadores de alcance, uma vez que estes permitem verificar como se saberia que se está fazendo a diferença. Considera-se que a avaliação do ambiente externo e seus impactos são aspectos relevantes, lembrados com muita propriedade pelo autor, e que não deve ser minimizado, uma vez que 'fazer a diferença' torna-se condição sine qua non à sobrevivência, no atual mundo globalizado e competitivo.

As Normas Internacionais ISO 11620 (ISO, 1998) divulgam, ainda, critérios para a aplicação de indicadores. Estes critérios, comentados por Gómez Hernández (2002), encontram eco nos estabelecidos por Geisler (2000). Para estes autores, ao se utilizar indicadores em sistemas e serviços de informação deve-se assegurar de que o indicador reúna as seguintes características: 
a) conteúdo informativo, ou seja, se o indicador aporta informação esclarecedora, de tal forma que se mostre útil para medir uma atividade, identificar os sucessos obtidos, localizar problemas ou deficiências para atuar na sua prevenção ou remédio. Deve auxiliar na tomada de decisão, na fixação das metas e prioridades;

b) confiabilidade, indicando que ele é confiável, a medida que produz o mesmo resultado sempre que se apresentam as mesmas circunstâncias. O fato de um indicador refletir a variabilidade implícita dos dados, por exemplo, as variações sazonais ou as flutuações nas atividades de empréstimo, não significa que não seja confiável;

c) validade, expressando que o indicador deve medir o que se quer medir;

d) idoneidade, na medida em que deve adequar-se ao objetivo para o qual foi formulado. As unidades e escala devem ser adequadas e as operações necessárias para implantar o processo de medida precisam ser compatíveis com os procedimentos habituais dos sistemas e serviços de informação documental;

e) praticidade, ao se referir ao fato de que o indicador deverá utilizar dados acessíveis, com um esforço razoável, em termos de tempo e de grau de capacitação do pessoal, custos operativos, além do tempo e paciência requeridos aos usuários;

f) comparabilidade, ou seja, se o indicador é utilizado para comparar unidades e serviços de informação documental, ele deve permitir que isto aconteça. Tal fato ocorre se, uma vez levados em conta os possíveis defeitos de exatidão, o mesmo resultado obtido significa que o nível de qualidade de serviços ou de deficiência, das unidades e serviços de informação, comparados, é o mesmo.

Outro aspecto a ser salientado, e que também é comentado pelos autores consultados, diz respeito às limitações dos indicadores. Neste sentido, deve-se estar atento ao fato de que os sistemas e serviços de informação não podem, sistematicamente, obterem valores ótimos em uma mesma vez, em todos os indicadores. Não é possível, por exemplo, alcançar-se um alto nível de satisfação de usuários com um custo, por usuário, muito baixo. Desta forma, os resultados devem ser interpretados com precaução, assegurando-se que não tenha havido erros na amostra ou subjetividade nas medições.

Gómez Hernández (2002) comenta que, às vezes, o comportamento dos usuários também influi e cita como exemplo o fato de que uma satisfação baixa com a disponibilidade de materiais pode significar o resultado de uma falta de conhecimento, por parte dos usuários, 
em localizar os documentos e não uma insuficiência destes documentos. No entanto, convém sublinhar, que sempre um valor baixo, ressaltado pelo indicador, denota que é necessário revisar o funcionamento na área em que tal valor foi obtido.

\section{CONCLUSÕES}

Sejam quais forem as necessidades ou as características de cada organização, a avaliação de serviços é fundamental para o planejamento, como auxiliar na tomada de decisão e, principalmente, para a gestão da qualidade. Sem qualidade, corre-se o risco de se perder o cliente/usuário. Como enfatiza Sutter (2002), o cliente é o coração das preocupações de qualquer organização. A satisfação do cliente deve ser o fio condutor da gestão da qualidade. O mesmo autor especifica, referindo-se ao parágrafo 8.5 da Norma ISO 9001 (ABNT, 2000), que, em matéria de gestão da qualidade, a organização deve planejar e gerar os processos necessários à melhora contínua do sistema de gestão da qualidade, conduzindo ações corretivas para eliminar as causas de não conformidade, a fim de impedir seu reaparecimento e identificar ações preventivas, permitindo eliminar as causas potenciais de não conformidade, para evitar que elas sobrevivam. Sutter (2002) reconhece que indicadores são ferramentas, de uso reconhecido internacionalmente, que possibilitam esse planejamento e gestão, favorecendo a comparação de dados.

A importância e a necessidade de se adotar indicadores para a avaliação de recursos de informação é inegável. O conhecimento de documentos que tratam de indicadores permite entender melhor o que eles significavam para as instituições ligadas à informação e ao conhecimento, como eles podem ser construídos, quais as metodologias mais utilizadas e adaptáveis ao processo e, principalmente, que indicadores, já testados, encontram-se disponíveis para compor o conjunto de indicadores a serem utilizados ou construídos na mensuração de desempenho de unidades e serviços de informação.

A informação, per si, é a fonte primordial da geração e apropriação do conhecimento. As unidades e serviços de informação devem preocupar-se em possuir competência para proporcionar a geração e a apropriação do conhecimento, focando suas ações na satisfação do cliente/usuário, seja este uma pessoa ou uma organização. Na busca pela constante adequação e excelência de seus serviços e produtos às demandas e necessidades de seus clientes/usuários, os indicadores surgem como uma valiosa ferramenta de gestão, ainda que pouco empregada. Seu uso sistemático aponta os pontos fortes e as debilidades da 
organização, bem como permite a comparação, tanto da organização com ela mesma, em diferentes momentos, como dela com outras organizações, facilitando o benchmarking, por exemplo.

Para reverter este quadro de desconhecimento ou desábito do uso sistemático de indicadores, duas ações são sugeridas: a sensibilização de órgãos de pesquisa e organismos governamentais quanto à adoção e incentivo ao seu uso; a implementação, junto aos cursos ligados à área de Ciência da Informação, em todos os níveis acadêmicos, de conteúdos sobre o tema, dentro da perspectiva de desenvolvimento da competência profissional.

\section{REFERÊNCIAS}

ASSOCIAÇÃO BRASILEIRA DE NORMAS TÉCNICAS-ABNT. NBR ISO 9001; sistemas de gestão da qualidade - requisitos: ABNT, dez.2000.

ALBORNOZ, Mario; FERNÁNDEZ POLCUCH, Ernesto. Indicadores en CyT: reencuentro de la política con la gestión, 1997. Disponível em: $<$ http://rucyt.edu.ar $>$. Acesso em: 01 jun.2003.

BABA, Dato'Zawiyah. Performance indicators for national libraries in Asia/Oceania: preliminary proposals based on a survey of Asia/Oceania libraries. In: IFLA GENERAL CONFERENCE, 69, Berlin, 2003. Electronic Proceedings ... IFLA, 2003. Disponível em: $<$ www.ifla.org/IV/ifla69/papers/025e-Baba_Shukor.pdf >. Acesso em: 26 maio.2005.

BRIAND, Bénédicte; BUFFETEAU, Annie; CUDELOU, Jean-François; DROUET, Claire; MANGETO, Annie; PAILLARD, Marie-Danièle; REGNAULT, Madeleine. Indicateurs de Perfomance des Services Documentaires; l'expérience d'un groupe de professionnels de l'information. Documentaliste - Sciences de l'Information, Paris, v. 39, n. 1-2, p.26-33, 2002.

BROPHY, Peter. Performance Measures for the 21st Century Libraries, [2000?].

Disponível em. < http://www.arl.org/pubscat/pubs/northumbria.htm> e $<$ http://www.arl.org/stats/north/video.html $>$. Acesso em: 26 nov.2002.

; CLARKE, Zoë. Equinoccio: medida de funcionamiento de la biblioteca y sistema de gerencia de la calidad, 2001. Disponível em: $<$ http://equinox.dcu.ie $>$. Acesso em: 16 nov.2002.

; CLARKE, Zoë; BRINKLEY, Monica; MUNDT, Sebastian; POLL, Münster Roswita. EQUINOX; Library Performance Measurement and Quality Management System.Performance Indicators for Electronic Library Services, 2000. Disponível em: <http://equinox.dcu.ie/reports/pilist.html\#intro>. Acesso em: 16 nov.2002.

FIINANCIADORA DE ESTUDOS E PROJETOS- FINEP. Termos e Conceitos. Rio de Janeiro, 2002. Disponível em: <http://www.finep.gov.br/empresa/conceitos_ct.asp?>. Acesso em: 22 ago.2002. 
FRANCISCO, Lourdes Terezinha dos Santos; FRACASSO, Edi Madalena. Indicadores para Avaliação de Resultados de Projetos de Interação Universidade-Empresa.

[2000?]. Disponível em. <http://nitec.ea.ufrgs.br/prof_edi2.htm>. Acesso em: 24 ago.2002.

GEISLER, Eliezer. The Metrics of Science and Technology. Connecticut: Quorum, 2000 .

GÓMEZ HERNÁNDEZ, J.A.. Gestión de Bibliotecas: la planificación y el marketing. In: GÓMEZ HERNÁNDEZ, J.A. Gestión de Bibliotecas. Murcia, 2002. (Gestión de Bibliotecas: la planificación y el marketing da Série). Disponível em:

$<$ http://gti1.edu.um.es:8080/jgomez/bibgen/intranet/03gestiona.PDF>. Acesso em: 24 ago.2002.

INTERNATIONAL FOR ORGANIZATION STANDARDIZATION - ISO. ISO

11620:1998(F); Information et Documentation - Indicateurs de performance des bibliothèques. Genebra: ISO, 1998.

. ISO 11620:2003(F); Information et Documentation -

Indicateurs de performance des bibliothèques. Emenda 1: indicadores complementares de performance das bibliotecas. Genebra: ISO, 2003a.

ISO/TR 20983:2003(E); Information and Documentation -

Performance Indicateurs for eletronic library services. Genebra: ISO, $2003 \mathrm{~b}$.

MARTINEZ, Eduardo; ALBORNOZ, Mario. Indicadores de Ciência y Tecnologia; estado del arte y perspectivas. Caracas: UNESCO, 1998.

MOORE, Nick. Comment Mensurer l'Efficacité des Bibliothèques Publiques; project de manuel. Paris: UNESCO, 1989.

ORGANISATION DE COOPÉRATION ET DE DÉVELOPPEMENT ÉCONOMIQUES OCDE. Glossary of Statistical Terms. Genebra: OCDE, 2001. Disponível em:

$<$ http://cs3-hq.oecd.org/scripts/stats/glossary/detail.asp?ID=2547>. Acesso em: 07 jan. 2002.

SUTTER, Éric. Documentation, Information , Connaissances: la gestion de la qualité. Paris: ABFF 2002. (Collection Sciences de l'Information. Série Ètudes et Techiniques).

. La Démarche Qualitée Appliqué aux Bibliothèques et Services

d'Information; questions-responses. Bulletin des Bibliothèques de France, Villeurbanne, n. 1, 1998. Disponível em: <http://enssibhp.enssib.fr8080/Enssib/bbf/bbf-98-1/02-

Sutter.pdf>. Acesso em: 13 dez.2002.

WILSON, Anne; PITMAN, Leeanne; TRAHN, Isabella. Guidelines for the Best Practice in Australian Univesity Libraries; intranational and international benchmarks. Canberra: Commonwealth, 2000. Disponível em:

$<$ http://www.dest.gov.au/archive/highered/eippubs/eip00_11/00_11.pdf $>$. Acesso em: 01 maio.2003. 


\section{Helen Beatriz Frota Rozados}

Profa. Adjunta do Departamento de Ciências da Informação da Faculdade de Biblioteconomia e Comunicação da Universidade Federal do Rio Grande do Sul (DCI/FABICO/UFRGS)

Bibliotecária CRB 10/368

Dra. em Comunicação e Informação.

hrozados@portoweb.com.br

Artigo aceito para publicação em: 07/ 2005 